DOI: http://dx.doi.org/10.12957/demetra.2015.13182

\title{
Conhecimento e abordagem sobre alimentação saudável por professores do ensino fundamental
}

\section{Knowledge and approach to healthy eating by primary education teachers}

\author{
Kátia Francielly Bezerra ${ }^{1}$ \\ Laura Caroline Ferreira Mendes Capuchinho' \\ Lucinéia de Pinhol,2 \\ ' Faculdade de Saúde Ibituruna, Departamento \\ de Nutrição. Montes Claros-MG, Brasil.

${ }^{2}$ Universidade Estadual de Montes Claros,
Mestrado Profissional em Cuidado Primário.
Montes Claros-MG, Brasil.
Correspondência / Corrrespondence
Lucinéia de Pinho
Campus Universitário Professor Darcy Ribeiro,
Vila Mauriceia. Montes Claros, MG, Brasil
CEP 39.401-089
E-mail: lucineiapinho@hotmail.com

\section{Resumo}

Avaliaram-se o conhecimento e a abordagem em alimentação saudável de professores de escolas públicas municipais urbanas de Montes Claros-MG. Com abordagem descritiva e quantitativa, entrevistaram-se 158 docentes de 28 escolas no ano de 2013. Os professores responderam um questionário com questões abertas referente à abordagem do tema "alimentação saudável" (QAAS); e outro com questões de múltipla escolha sobre o "conhecimento nutricional e estratégias para alimentação saudável” (QN). Dos 12 temas do QAAS, sete não foram abordados pela maioria dos professores, que de modo geral não exploraram metodologias alternativas de ensino. O ponto positivo destacado pelos professores foi a aprendizagem dos alunos; os aspectos negativos foram falta de material didático e influência negativa da família. O QN mostrou que $73 \%$ dos professores não receberam capacitação específica para trabalhar o tema, e que 72,15\% tinham conhecimento moderado dos conteúdos. Conclui-se que as metodologias e recursos didáticos adotados para o ensino da alimentação saudável na escola ainda são incipientes, tímidos e pouco explorados. Ainda, o conhecimento dos professores é apenas moderado, indicando que eles deveriam receber capacitação específica em alimentação saudável e nutrição.

Palavras-chave: Alimentação Escolar. Educação Alimentar e Nutricional. Promoção em Saúde. 


\section{Abstract}

The study evaluated the knowledge and approach in heathy eating of teachers of urban municipal public schools in Montes Claros-MG, Brazil. In a descriptive and quantitative approach, 158 teachers from 28 schools were interviewed in 2013. They answered a questionnaire with open questions regarding the approach to health eating (QAAS); and other with multiple choice questions on knowledge on nutrition and strategies for healthy eating $(\mathrm{QN})$. From the 12 topics contained in the QAAS, 7 were not addressed by most teachers, who in general seldom adopted alternative teaching methodologies. The positive point highlighted by the teachers was student progress; the negative aspects were lack of didactic material and negative influence of the family. The QN showed that $73 \%$ of the teachers did not receive specific training to address the issue, and that $72.15 \%$ showed moderate knowledge on it. In conclusion, the methodologies and didactic resources adopted in health eating teaching at school are still incipient, timid and poorly exploited. Moreover, the knowledge of the teachers is only moderate, indicating that they should undergo specific training programs on health eating and nutrition.

Key words: Student Eating. Eating and Nutrition Education. Health Promotion.

\section{Introdução}

A alimentação saudável é um hábito que deve ser ensinado e praticado desde a infância, pois quanto mais cedo for adotado, maiores as chances de ser continuado em fases posteriores da vida. ${ }^{1,2}$ Assim, desde seus primeiros anos de vida, o indivíduo deveria receber sistematicamente informações abrangentes sobre qualidade e tipos de alimento e suas preparações. ${ }^{3}$

A promoção da alimentação saudável na escola é uma estratégia importante no âmbito da nutrição, visto que as crianças passam muitas horas nesse ambiente. Além de orientar a prática de hábitos saudáveis, o ambiente escolar tem potencial para possibilitar a contextualização do conhecimento no universo dos estudantes, através de uma abordagem integral e contextualizada dentro da realidade familiar, comunitária e social. ${ }^{4}$ Nesse sentido, mais que prover informações, as disciplinas escolares ajudam no desenvolvimento de valores relacionados ao estilo de vida saudável. ${ }^{2,5}$ 
A atuação dos professores como fonte de informação e elemento de comunicação é imprescindível no ambiente escolar. Para que atuem de modo eficiente na promoção da saúde, é fundamental que esses profissionais possuam informações atualizadas sobre saúde, independentemente das disciplinas que ministram, e que apliquem o conteúdo de maneira transversal e interdisciplinar em suas práticas pedagógicas. ${ }^{5-7}$ Além disso, é importante que saibam fazer uso de recursos pedagógicos apropriados, ${ }^{8}$ a fim de promover não apenas o conhecimento sobre hábitos alimentares saudáveis, mas que também influenciem a atitude e a prática dos mesmos entre os escolares. ${ }^{2}$

Um aspecto a ser considerado é que o domínio de conteúdo por parte dos professores não é suficiente para que ele desempenhe sua função de educador na área de saúde; é necessário que eles reconheçam a relevância da sua atuação, ${ }^{4}$ pois mais que se envolverem na realidade social, os professores podem servir de referência de conhecimento e comportamento para os alunos. ${ }^{6,9,10}$ Nesse sentido, para o trato adequado de temas de educação em saúde e obtenção de resultados práticos satisfatórios, é importante que os professorem recebam orientação de qualidade. A preparação deve ser baseada na prática dos educadores, isto é, a forma como abordam os temas de saúde no ambiente escolar.

No intuito de subsidiar planos que melhorem o trabalho de temas de saúde em sala de aula, foram avaliados o conhecimento em "alimentação saudável" de professores de escolas públicas municipais de Montes Claros-MG, e a forma como o abordam em sala de aula.

\section{Metodologia}

Trata-se de estudo transversal, realizado no período de agosto a outubro de 2013, em 28 escolas urbanas da rede de ensino público municipal de Montes Claros, Estado de Minas Gerais. Conforme dados da Secretaria Municipal da Educação, no período do estudo trabalhavam na rede 525 professores efetivos e 115 contratados, sendo que 550 estavam na regência de classes.

A população-alvo consistiu de professores dos cinco primeiros anos (séries) do ensino fundamental que estavam em regência de classe. A partir de uma lista obtida da Secretaria Municipal de Educação, selecionaram-se os profissionais a serem avaliados por amostragem aleatória simples. Estimou-se uma amostra mínima de 182 profissionais, considerando uma margem de erro de $5 \%$, nível de confiança de $90 \%$ e frequência do evento esperado de $50 \%$. 
Os critérios de inclusão foram: responder os questionários em pelo menos uma de três tentativas de abordagem, concordar com a pesquisa e assinar o Termo de Consentimento Livre e Esclarecido de participação. Foram excluídos do estudo professores de alunos de escolas especiais, ensino religioso, em licença ou férias. Foram excluídos também professores de educação física porque, embora estejam aptos para trabalhar temas de alimentação, na prática priorizam a atividade física.

Os professores responderam a um Questionário Estruturado sobre Abordagem do tema Alimentação Saudável (QAAS), ${ }^{11}$ que investigou os conteúdos ministrados em sala de aula, metodologias de abordagem, êxito da ação e sua capacitação. Os temas elencados no QAAS foram extraídos da coleção "Conhecer e Crescer", edição 2013, adotada pela rede de ensino Municipal de Montes Claros, do 1ํa a $5^{\underline{0}}$ ano escolar. Trabalhando os conteúdos de modo interdisciplinar e contextualizado, os temas abrangem desde a origem e composição dos alimentos até aspectos de higiene e saúde, como descrito na tabela 1 . As respostas em relação às metodologias e aspectos positivos e negativos das atividades educativas desenvolvidas foram descritivas, em questões abertas.

Aplicou-se também um questionário de Conhecimento Nutricional e Estratégias para Alimentação Saudável (QN), ${ }^{12}$ contendo questões de múltipla escolha que avaliaram: conhecimento acerca de gorduras boas ou ruins, alimentação saudável, práticas alimentares saudáveis, conteúdo de sal nos alimentos e doenças relacionadas à alimentação. O nível de conhecimento nutricional foi medido a partir do número acertos por domínio, sendo classificado como baixo (abaixo de $60 \%$ ), moderado (entre 61 e $80 \%$ ) e alto (acima de $81 \%$ ).

As entrevistas foram realizadas nas escolas, em horário de aula. Os questionários QN e QAAS foram entregues aos professores e recolhidos três dias após. Os dados foram analisados por estatística descritiva, utilizando-se o programa SPSS versão 18.0.

O estudo obedeceu aos preceitos éticos para pesquisa com seres humanos, conforme disposto na Resolução no 196/96, do Conselho Nacional de Saúde do Brasil. O protocolo experimental foi aprovado pelo Comitê de Ética da SOEBRAS (Associação Educativa do Brasil / Faculdades Unidas do Norte de Minas), processo no. 387.524. A aplicação do questionário foi autorizada pelas escolas, e os entrevistados estavam cientes de que poderiam desistir da participação a qualquer momento.

\section{Resultados}

Foram entrevistados 158 professores (taxa de resposta $=86,81 \%$ ), $98 \%$ do sexo feminino e apenas $2 \%$ do sexo masculino. A maioria dos professores tinha entre 38 e 47 anos de idade (50,63\%), 23,42\% abaixo e $19,95 \%$ acima dessa faixa etária. 
Na resposta ao QAAS, verificou-se que, embora todos os temas elencados a partir do livro didático (tabela 1) tenham sido citados por pelo menos um professor, os conteúdos não foram desenvolvidos por todos os professores. Os mais trabalhados foram "a importância da alimentação", "higiene dos alimentos" e "o que é uma boa alimentação". Porém, dos 12 temas, sete não foram abordados pela maioria dos professores, e mais de 60\% não discutiram "vitaminas e minerais", "grupos alimentares (pirâmide alimentar)", "nada de exageros", "alimentos fornecedores de energia", "alimentos construtores" e "carência e excesso de alimento".

Tabela 1. Temas em Alimentação Saudável propostos no material didático e abordados em sala de aula. Montes Claros-MG, 2013.

\begin{tabular}{lcccc}
\hline \multicolumn{1}{c}{ Temas } & \multicolumn{2}{c}{ SIM } & \multicolumn{2}{c}{ NÃO } \\
\hline A importância da alimentação & $\mathrm{n}^{*}$ & $\%$ & $\mathrm{n}^{*}$ & $\%$ \\
O que é uma boa alimentação & 149 & 94,30 & 09 & 5,70 \\
Vitaminas e minerais & 131 & 82,90 & 27 & 17,10 \\
Solo fértil, alimento mais saudável & 63 & 39,87 & 95 & 60,13 \\
Alimentos bem nutritivos & 68 & 43,04 & 90 & 56,96 \\
Higiene dos alimentos & 76 & 48,10 & 82 & 51,90 \\
De onde vêm os alimentos & 132 & 83,54 & 26 & 16,46 \\
Grupos alimentares (pirâmide) & 95 & 60,13 & 63 & 39,87 \\
Nada de exageros & 51 & 32,28 & 107 & 67,72 \\
Alimentos fornecedores de energia & 47 & 29,75 & 111 & 70,25 \\
Alimentos construtores & 30 & 18,99 & 128 & 81,01 \\
Carência e excesso de alimento & 45 & 28,48 & 113 & 71,52 \\
\hline
\end{tabular}

Ainda no QAAS, os professores indicaram variar as metodologias para trabalhar os temas junto aos alunos (tabela 2), mas que declararam adotar principalmente pesquisa em livros, jornais e revistas e aulas expositivas. Outras metodologias, como recursos visuais e recursos auditivos, foram pouco usadas. 
Tabela 2. Metodologias adotadas para abordagem do tema "alimentação e nutrição" em sala de aula. Montes Claros-MG, 2013.

\begin{tabular}{|c|c|c|}
\hline Metodologia & n (158) & $\%$ \\
\hline Recursos visuais & 01 & 0,63 \\
\hline Cartazes e murais & 01 & 0,63 \\
\hline Alimentação escolar (merenda) & 01 & 0,63 \\
\hline teatro & 02 & 1,26 \\
\hline Recursos Auditivos & 02 & 1,26 \\
\hline Elaboração de receitas & 02 & 1,26 \\
\hline Exposição e análise de rótulos de alimentos & 05 & 3,16 \\
\hline Trabalho em equipe & 05 & 3,16 \\
\hline Brincadeiras & 05 & 3,16 \\
\hline Dinâmica & 06 & 3,80 \\
\hline Internet/informática & 09 & 5,70 \\
\hline Atividades práticas e degustação & 11 & 6,96 \\
\hline Vídeos e filmes & 19 & 12,03 \\
\hline Debates e roda de conversas & 26 & 16,46 \\
\hline Pesquisa em livros, revistas e jornais & 55 & 34,81 \\
\hline Livro didático & 27 & 17,09 \\
\hline Resolução de atividades em sala & 32 & 20,25 \\
\hline Aula dialogada & 34 & 21,52 \\
\hline Aula expositiva & 43 & 27,22 \\
\hline Palestras & 03 & 1,90 \\
\hline Entrevistas & 04 & 2,53 \\
\hline
\end{tabular}

Os professores levantaram, também no QAAS, 12 aspectos positivos (tabela 3) e 14 aspectos negativos (tabela 4) das atividades desenvolvidas. Entre os positivos, destacam-se "aprendizado e conhecimento adquirido", seguido de "interesse"; e entre os negativos, "falta de material didático" e "influência negativa da família".- 
Tabela 3. Pontos positivos no trabalho de temas relacionados à alimentação e nutrição em sala de aula. Montes Claros-MG, 2013.

\begin{tabular}{lcc}
\hline \multicolumn{1}{c}{ Pontos } & $\mathrm{N}(158)$ & $\%$ \\
\hline Interesse & 22 & 13,92 \\
Participação e envolvimento & 09 & 5,70 \\
Curiosidade & 05 & 3,16 \\
Conhecimento prévio & 06 & 3,80 \\
Conscientização & 17 & 10,76 \\
Facilidade de compreensão das crianças & 05 & 3,16 \\
Descobertas importantes & 01 & 0,63 \\
Reflexão sobre o assunto & 03 & 1,90 \\
Mudança de hábitos alimentares e higiênicos & 21 & 13,29 \\
Aprendizado e conhecimento adquirido & 40 & 25,32 \\
Influência da alimentação escolar & 02 & 1,26 \\
Mudança na instituição (acabou com a vendinha) & 01 & 0,63 \\
\hline
\end{tabular}

Tabela 4. Pontos negativos no trabalho de temas relacionados à alimentação e nutrição em sala de aula. Montes Claros-MG, 2013.

\begin{tabular}{lcc}
\hline \multicolumn{1}{c}{ Pontos } & $\mathrm{n}(158)$ & $\%$ \\
\hline Objetivos não alcançados & 2 & 1,27 \\
Influência negativa da família & 27 & 17,09 \\
Resistência a mudanças de hábitos & 8 & 5,06 \\
Falta de conhecimento prévio & 2 & 1,27 \\
Falta de conhecimentos dos alunos e familiares & 3 & 1,90 \\
Falta de material didático & 16 & 10,13 \\
Falta de interesse do aluno & 3 & 1,90 \\
Falta de acesso à alimentação & 2 & 1,27 \\
Influência da indústria de alimentos & 12 & 7,59 \\
Falta de capacitação para os docentes & 6 & 3,80 \\
Incoerência da alimentação escolar (merenda) com o & & \\
que se ensina & 3 & 1,90 \\
Condição socioeconômica do aluno & 12 & 7,59 \\
Não existiram pontos negativos & 62 & 39,24 \\
\hline
\end{tabular}


Em relação à preparação para trabalhar os temas relacionados à alimentação e nutrição em sala de aula, 117 professores $(74 \%)$ disseram não ter recebido capacitação específica-

Nas respostas ao QN (tabela 5), observou-se maior nível de acertos dos professores no domínio referente ao conhecimento sobre "conteúdo de sal nos alimentos". Em uma análise geral, dos 158 professores entrevistados, nove (5,7\%) mostraram baixo nível de conhecimento; 35 (22,15\%), alto conhecimento; e 114 (72,15\%), conhecimento moderado.

Tabela 5. Acertos do questionário sobre conhecimento nutricional e estratégias para uma alimentação saudável, aplicado a professores de ensino fundamental. Montes Claros-MG, 2013.

\begin{tabular}{lccc}
\hline \multicolumn{1}{c}{ Domínios } & $\begin{array}{c}\text { Indivíduos que } \\
\text { acertaram as } \\
\text { questões }(\mathrm{n}=158)\end{array}$ & $\%$ & $\begin{array}{c}\text { Nível de } \\
\text { conhecimento }\end{array}$ \\
\hline Conhecimento em gordura trans & 115 & 72,78 & Moderado \\
$\begin{array}{l}\text { Percepção de alimentação saudável } \\
\text { Conhecimento sobre práticas }\end{array}$ & 117 & 74,05 & Moderado \\
alimentares saudáveis & 122 & 77,22 & Moderado \\
$\begin{array}{l}\text { Conhecimento sobre conteúdo de sal } \\
\text { Conhecimento sobre doenças }\end{array}$ & 140 & 88,61 & Alto \\
relacionadas à alimentação & 106 & 67,09 & Moderado \\
\hline \multicolumn{1}{c}{ Média } & 120 & 75,95 & Moderado \\
\hline
\end{tabular}

\section{Discussão}

As ações em saúde geralmente seguem o modelo biomédico tradicional, isto é, são predominantemente voltadas à lógica da prevenção e da recuperação da saúde. ${ }^{13}$ No entanto, ações de promoção da saúde, segurança alimentar e nutricional, ${ }^{13}$ tais como educação alimentar, podem ser ferramentas valiosas para fortalecer políticas públicas de saúde, respeitando os aspectos socioculturais do público-alvo. ${ }^{14}$ Quando esse público consiste de escolares, o professor é um elemento chave para o sucesso da ação, uma vez que é capaz de contextualizar os conteúdos no cotidiano do educando. No entanto, como mostrado no presente estudo, a atuação desse profissional é ainda restrita. A implementação da educação nutricional ainda requer esforços de capacitação de professores, a fim de habilitá-los para trabalhar com alternativas metodológicas mais dinâmicas e atualizar os conhecimentos específicos, particularmente na rede pública e municipal de ensino de Montes Claros-MG. 
No presente estudo, pouco mais da metade da população tinha entre 38 e 47 anos de idade, revelando um grupo maduro e dentro da faixa etária do município, que segundo a Secretaria de Educação, varia de 18 a mais que 58 anos. Os temas por eles trabalhados constavam da coleção didática do $2^{\underline{0}}$ e $3 \underline{0}$ anos do ensino fundamental, mas não faziam parte do currículo obrigatório. Essa flexibilidade curricular pode explicar por que mais da metade dos professores entrevistados abordaram apenas quatro dos 12 temas elencados. Os resultados da tabela 1 sugerem que temas mais comuns, como "o que é a boa alimentação", "a importância da alimentação" e "a higiene dos alimentos", sejam mais abordados que temas mais específicos como "o que são alimentos construtores" ou "a pirâmide alimentar".

Apesar de não serem obrigatórios, os conteúdos de nutrição estão inseridos como assuntos transversais no tema saúde dos Parâmetros Curriculares Nacionais (PCNs). Os PCNs constituem uma proposta ainda inovadora e flexível, de caráter integrado e promotora de cidadania. ${ }^{15}$ Como subcategoria do PCN, o conteúdo de Nutrição é muitas vezes trabalhado informalmente em sala de aula, sem o registro das atividades desenvolvidas ou avaliação dessas práticas. Porém, dada sua importância, alguns autores defendem sua inclusão nos conteúdos exigidos no ensino fundamental. Essa medida requer investimentos de implantação e atendimento de requisitos técnico-científicos fundamentais,${ }^{16}$ mas em contrapartida confere legitimidade e reforça a necessidade de abordagem desses conteúdos pelos educadores. ${ }^{17}$

A orientação nutricional pode ser ministrada por metodologias variadas, como exposições orais, dinâmicas em grupo, leitura dirigida, experiências práticas, utilização de vídeos, filmes e dramatização. ${ }^{18}$ No presente estudo, os professores trabalharam o assunto principalmente por meio de pesquisa (livros, revistas e jornais), aulas dialogadas e resolução de atividades em sala de aula, mas usaram também métodos tradicionais como aula expositiva.

Percebe-se que a diversificação na metodologia adotada pelos professores entrevistados foi ainda tímida. Por exemplo, recursos de apoio como material audiovisual não deveriam ser meros coadjuvantes do aprendizado; eles devem ser provocativos e não meramente contemplativos, e com expressiva ênfase em imagens e esquemas didáticos que levem a pensar e não a imprimir conhecimento pronto. ${ }^{16}$ Essa ideia condiz com a proposta da metodologia ativa, que se baseia no princípio de que a criança é um ser em desenvolvimento e cujo crescimento físico e intelectual se dá através de sua atividade espontânea e natural, a qual pode ser orientada pelo professor para novas descobertas..$^{19} \mathrm{O}$ próprio material didático da coleção "Conhecer e Crescer" leva ao desenvolvimento dessa metodologia, uma vez que propõe trabalhar os temas de forma contextualizada e integrada com a realidade dos educandos. Mas quaisquer que sejam as estratégias adotadas, o importante é que sejam planejadas adequadamente para o atendimento dos objetivos esperados. ${ }^{18}$ 
O ensino de nutrição deveria ser incorporado à educação fundamental com metodologias que respeitem algumas premissas pedagógicas, tais como: 1) diálogo com o aluno, de modo a levar a coordenação de ações e não apenas em transmissão de informações; 2) conteúdo que faça sentido para os alunos; 3) educação problematizadora, que leva à reflexão sobre causas, mecanismos e soluções das questões nutricionais; 4) abordagem transversal, integrada com as demais matérias do currículo; 5) atividades lúdicas, motivadoras e mediadoras da compreensão do real pela criança; 6) ensino construtivista; e 7) cultivo da construção de cidadania, pelo fomento de senso crítico e empreendedor. ${ }^{16}$ Além disso, o educador precisa sentir-se bem em sala de aula e melhorar sua comunicação com os alunos, aumentando seu potencial para exercer ensino e aprendizagem. É preciso estabelecer primeiro uma relação empática entre educador e estudantes, buscando mapear interesses, perspectivas, expectativas e outros, para então descobrir as competências dos estudantes e as contribuições que cada um pode dar no processo de aprendizagem. ${ }^{14}$

Associado à falta de dinâmica na aplicação das aulas, os professores entrevistados relataram enfrentar falta de material didático sobre educação nutricional. Sendo esse um assunto transversal no programa escolar, não é necessário que se elabore um projeto fechado para sua aplicação, mas é preciso que sejam delineadas, de forma clara, diretrizes mestras nas quais o educador possa se pautar para desenvolver os conteúdos pertinentes. ${ }^{14}$ Nesse sentido, o Fundo Nacional de Desenvolvimento da Educação (FNDE) estabeleceu, em suas normas para a execução do Programa Nacional de Alimentação Escolar (PNAE), diretrizes que incluem a educação alimentar e nutricional no processo de aprendizagem que perpassa o currículo escolar, abordando o tema da alimentação e nutrição e o desenvolvimento de práticas saudáveis de vida na perspectiva da segurança alimentar e nutricional. ${ }^{20}$ Embora isso já represente um grande avanço, o desenvolvimento e a disponibilização de recursos didáticos dinâmicos são ainda necessários para subsidiar a ação do professor.

Os professores mencionaram outro ponto negativo relevante no que diz respeito à influência negativa da família nos hábitos alimentares dos estudantes. Isso mostra que a escola e a família trabalham em direções opostas, isto é, enquanto a escola indica o ideal de alimentação saudável, a realidade do aluno é outra. O papel da família na alimentação e na educação nutricional das crianças, porém, é inquestionável, visto que ela seria responsável pela concretização do conteúdo aprendido. ${ }^{21}$ Como pode abstrair-se das percepções dos professores e corroborando outros estudos, as atividades para a promoção da alimentação saudável devem ser direcionadas para toda a comunidade escolar, incluindo alunos, professores e pais. ${ }^{16}$

A despeito dos hábitos alimentares familiares, uma alternativa para a prática da alimentação saudável seria a incorporação da alimentação saudável no ambiente escolar. A boa alimentação promove a educação nutricional no âmbito da escola, de forma a reforçar a aquisição de bons hábitos alimentares, e também contribui para o melhor desempenho escolar, reduzindo a evasão e a repetência escolar, conforme objetivos do Programa Nacional de Alimentação Escolar (PNAE). ${ }^{20}$ Há que se considerar, porém, que o professor, que seria um modelo para os alunos, não é 
obrigatoriamente um exemplo positivo de saúde no ambiente escolar. É comum que os professores comprem com recursos próprios os lanches para os intervalos entre aulas e enfrentem, nas escolas, restrições das mais diversas ordens para que tenham uma alimentação que possa ser considerada "saudável". Outros estudos mostram que, quando professores preferem alimentos industrializados à merenda escolar saudável, os alunos tendem também a consumir esses alimentos de menor qualidade nutricional. ${ }^{22}$ Nesse sentido, seria importante implementar medidas de proteção e ações regulatórias que impeçam a exposição de coletividades e indivíduos a fatores estimuladores de práticas não saudáveis, sobretudo por parte dos professores. ${ }^{22}$

Professores informados e motivados podem se tornar agentes transformadores do comportamento alimentar de escolares, ${ }^{22}$ mas para dominar o conhecimento é preciso que eles recebam capacitação específica. ${ }^{16}$ No entanto, os profissionais avaliados relataram carecer de preparo para discutir a alimentação saudável, sendo que maioria (74\%) relatou não ter recebido capacitação específica e mostrou ter conhecimento apenas moderado dos domínios avaliados (tabela 5), exceto pelo conhecimento sobre "consumo de sal”, que foi alto. O domínio dos conteúdos por parte dos professores é importante para melhorar sua atuação em programas de educação nutricional, ${ }^{23}$ e os resultados do presente estudo indicam que, de fato, os profissionais entrevistados têm uma fração significativa de conhecimento a ser adquirido.

Os programas de capacitação são fundamentais para preparar os professores para discutir "temas transversais", que cada vez mais são considerados importantes por atores sociais e que, a partir de seus pontos de vista, deveriam obrigatoriamente fazer parte das aulas. ${ }^{24}$ Dominar conteúdos e aplicá-los, fazendo uso de procedimentos sempre os mais criativos, críticos e reflexivos, de modo dinâmico e atraente, é um desafio que requer preparo numa realidade em que nem sempre a escola conta com professores para todas as matérias. ${ }^{25}$ De qualquer forma, os benefícios do engajamento em capacitação já foram mostrados em outros estudos que mostram exemplos significativos de melhora no conhecimento sobre alimentação e nutrição de escolares ensinados por professores que receberam orientação para o desenvolvimento de projeto educativo em educação nutricional no ambiente escolar., ${ }^{11,26}$

A capacitação não pode restringir-se a um evento pontual nem a um instrumento destinado a suprir deficiências da formação inicial; deve ser um programa contínuo, parte do exercício profissional do educador e que interrompa um círculo vicioso no qual não há professores treinados para o ensino de Nutrição, e os professores não são treinados por ausência ou falha dos cursos de formação específica. ${ }^{17}$ Essa capacitação deve ser provida por profissionais de saúde, com adaptações locais de conteúdos e objetivos, de modo a não apenas informar, como também sensibilizar os professores para o engajamento nessa atividade. ${ }^{16}$ A assessoria de um profissional capacitado não só permite a uniformidade da instrução, como também assegura ao educador o domínio do conteúdo ensinado, o que possivelmente favorecerá a compreensão e a assimilação prática de conteúdos por parte dos educandos..$^{11,26}$ 


\section{Considerações finais}

Seguindo a proposta do material didático usado nas escolas municipais de Montes Claros, a coleção "Conhecer e Crescer", os professores mostraram trabalhar com educação nutricional em sala de aula como tema transversal, isto é, incluído em diferentes programas e projetos educacionais. O desenvolvimento do tema é ainda baseado em recursos didáticos clássicos, como pesquisa em livros, jornais e revistas e aulas expositivas.

Os professores mostraram domínio apenas moderado da maioria dos conteúdos propostos no material didático. Reforçando isso, eles declararam não possuir conhecimentos específicos para se aprofundarem no tema da alimentação saudável. Considerando os resultados e o potencial do professor como agente promotor de saúde, sugere-se que programas locais de capacitação sejam elaborados, de modo a otimizar sua atuação nessa área.

\section{Referências}

1. Madruga SW, AraújoCLP, Bertoldi AD, Neutzling, MB. Manutenção dos padrões alimentares da infância à adolescência.Rev. Saúde Pública2012;46(2):376-386.

2. Brasil.Ministério da Saúde. Saúde na escola. Brasília: Ministério da Saúde;2009. Série B. Textos Básicos de Saúde Cadernos de Atenção Básica, n. 2.

3. Prado BG, Guimarães LV, Lopes MAL, Bergamaschi DP. Efeito de ações educativas no consumo de alimentos no ambiente escolar. Nutrire 2012;37(3): 281-292.

4. Yokota RTC, Vasconcelos TF, Pinheiro ARO, Schmitz BAS, Coitinho DC, Rodrigues MdLCF. Projeto a escola promovendo hábitos alimentares saudáveis: comparação de duas estratégias de educação nutricional no Distrito Federal, Brasil. Rev. Nutr.23(1):37-47.

5. HallalPC. Promoção da atividade física no Brasil: chegou a hora da escola. Revista Brasileiro de Atividade Física e Saúde2010; 15(2):76-77.

6. Oliveira LM. Promoção da saúde na educação física escolar: concepções e propostas na perspectiva de professores do ensino público estadual da região centro-oeste do município de São Paulo. Motriz 2010; 16(2):535-535.

7. Gaglianone CP, Taddei JAAC, Colugnati FAB, Magalhães CG, Davanço G, Macedo L, et al. Nutrition education in public elementary schools of São Paulo, Brazil: the reducing risks of illness and death in adulthood project. Rev. Nutr. 2006; 19(3):309-320.

8. Fernandez PM, Silva DO. Descrição das noções conceituais sobre os grupos alimentares por professores de $1^{a}$ a $4^{a}$ série: a necessidade de atualização dos conceitos. Ciênc.Educ.2008; 14(3):451-466.

9. Borges TT, Rombaldi AJ, Knuth AG, Hallal PC. Conhecimento sobre fatores de risco para doenças crônicas: estudo de base populacional. Cad. SaúdePública 2009; 25(7):1511-1520.

10. Doyle EI,Feldman RH. Are local teachers or nutrition experts perceived as more effective among 
Brazilian high school students? J. Sch. Health1994; 64(3):115-118.

11. Detregiachi CRP, Braga TMS. Projeto "criança saudável, educação dez": resultados com e sem intervenção do nutricionista. Rev. Nutr. 2011;24(1):51-59.

12. GuadagninSC. Elaboração e validação de questionário de conhecimentos em nutrição para adultos [dissertação]. Brasília: Faculdade de Ciências da Saúde, Universidade de Brasília; 2010.

13. Santos LAS.O fazer educação alimentar e nutricional: algumas contribuições para reflexão.Ciênc. Saúde Coletiva 2012; 17(2):455-462.

14. Brasil.Ministério da Saúde. Redenutri. Rede de nutrição do Sistema Único de Saúde. Brasília: Ministério da Saúde;2010. Texto de Sistematização 06: Educação alimentar e nutricional.

15. Brasil, Ministério da Educação e do Desporto. Parâmetros curriculares nacionais 1996. Brasília: MEC; 1996.

16. BizzoMLG, LederL. Educação nutricional nos parâmetros curriculares nacionais para o ensino fundamental. Rev. Nutr.2005; 18(5):661-667.

17. Brasil.Ministério da Saúde.Guia alimentar para a população brasileira: promovendo a alimentação saudável.Brasília: Ministério da Saúde; 2008. Série A.Normas e Manuais Técnicos.

18. PhilippiST. Educação nutricional e pirâmide alimentar. In: PhilippijRA, Pelicioni MCF, organizadores. Educação ambiental e sustentabilidade. São Paulo: Manole; 2005. p. 813-825.

19. PilettiN, PilettiC.História da educação. São Paulo: Ática; 1995.

20. Brasil.Fundo Nacional de Desenvolvimento da Educação. Portal do FNDE [Internet]. PNAE. Brasília: MEC; 2009.Disponível em: http://www.fnde.gov.br/

21. Marin T, Berton P, Santo LKRE. Educação nutricional e alimentar: por uma correta formação dos hábitos alimentares. RevF@pciência 2009; 3(7):72-78.

22. Fontes PG, Razuck RCSR, Razuck FB. A influência do professor nos hábitos alimentares. In: Anais do VII Encontro Nacional de Pesquisa em Educação em Ciências,Campinas; 2011.

23. Souza JA. Conhecimentos nutricionais, reprodução e validação do questionário [dissertação].Porto: Faculdade de Medicina, Universidade do Porto; 2009.

24. Brito AKA, Silva FIC, Franca NM, Nanci M. Programas de intervenção nas escolas brasileiras: uma contribuição da escola para a educação em saúde. Saúde Debate2012; 36(95):624-632.

25. Costa GMC, Cavalcanti VM, Barbosa ML, Celino SDM, França ISX, Sousa FS. Promoção de saúde nas escolas na perspectiva de professores do ensino fundamental. Rev. Eletr. Enf. 2013; 15(2):506-15.

26. Longo-Silva G, Taddei JAAC, Konstantyner T, Toloni MHA. Percepções de educadores de creches acerca de práticas cotidianas na alimentação de lactentes: impacto de um treinamento. Ciênc. Saúde Coletiva 2013; 18(2):545-552. 
